\title{
Editorial
}

\section{Network analysis and modelling: Special issue of European Journal of Applied Mathematics}

\author{
MASON A. PORTER ${ }^{1,2,3}$ and GINESTRA BIANCONI \\ ${ }^{1}$ Oxford Centre for Industrial and Applied Mathematics, Mathematical Institute, University of Oxford, Andrew \\ Wiles Building, Radcliffe Observatory Quarter, Woodstock Road, Oxford, OX2 6GG, UK \\ ${ }^{2} C A B D y N$ Complexity Centre, University of Oxford, Oxford, OX1 $1 \mathrm{HP}, \mathrm{UK}$ \\ ${ }^{3}$ Department of Mathematics, University of California, Los Angeles, California 90095, USA \\ email: mason@math.ucla.edu \\ ${ }^{4}$ School of Mathematical Sciences, Queen Mary University of London, London E1 4NS, UK \\ email: g.bianconi@qmul.ac.uk
}

(Received 13 July 2016; accepted 12 July 2016)

This special issue of the European Journal of Applied Mathematics features research articles on networks, one of the most exciting areas of applied mathematics.

The study of networks incorporates tools from a wide variety of areas-including graph theory (of course), dynamical systems, optimization, statistical physics, probability, statistics, and more - and is important for applications in just about any area that one might imagine. Scholars who study networks ask questions like the following: Who are the most important people and collaborations in a network of political committee memberships? How have ideas spread over Twitter and other social media in the \#Brexit debate, and how has this spread of ideas influenced opinions and events? What are the properties of entropy and random walks in networks with multiple subsystems or with multiple types of social ties? What is a good movie-recommendation strategy in a social network? Which parts of a granular material are the least stable, and how should one measure this? How can one improve transportation systems and building layouts to ease traffic congestion? How can one control cascading failures in infrastructure or financial networks? Which species are the most important ones in an ecosystem? What is the best vaccination strategy to contain a disease? Which sets of genes and molecular interactions are responsible for a given disease? Which molecular network properties explain the comorbidity of two diseases?

In its broadest form, a network is an abstraction of a complex system of interacting entities [17,21,24,31,33]. Most traditionally, a network is represented mathematically as a graph, with "nodes" encoding entities and "edges" encoding the interactions between those entities. However, the term "network" is more general than a graph, as a network can encompass connections among an arbitrary number of entities, can have nodes and/or edges that change in time, can include multiple types of edges, can have (and often do have) associated dynamical processes both on the network and of the network, and so on. Our special issue surveys a diverse cross section of topics in the mathematical 
study of networks - including a variety of methods and applications - but there are also many others. A few of the many other popular topics in network science are multilayer networks $[4,20]$, temporal networks [15,16], spatial networks [3], network inference [19], higher-order structures (e.g., simplicial complexes) in networks, percolation theory $[9,28]$, random graphs, visualization of networks, games on networks [18], and more. The applications of networks are even more diverse (and there are many review articles in a multitude of fields that focus on applications of networks to those fields), and we only touch on a few of them in this special issue.

Network analysis is a burgeoning area of both theoretical and applied mathematics. There are a large number of annual conferences (e.g., NetSci, Sunbelt, SIAM workshops, and many others) and journals that are devoted to networks, including an increasing number that focus on applications in specific disciplines. Propelled by theoretical advances and a deluge of data, the study of networks - as distinguished from more traditional mathematical subjects like graph theory-is playing an increasingly important role in industry, in government, and in mathematical and computational science departments at universities throughout the world. Network analysis is now recognized widely as one of the most important subjects in mathematics. A wealth of faculty positions devoted to networks and/or data science have been advertised in mathematics and applied mathematics departments over the last few years, and several new masters and doctoral programmes (including a few doctoral training centres in England) on complex systems and networks have been developed. Additionally, many mathematical institutes have had recent focal semesters on topics related to networks. Examples include a Fall 2016 semester programme on Theoretical Foundations of Statistical Network Analysis (https://www.newton.ac.uk/event/sna) at the Isaac Newton Institute for Mathematical Sciences, a Spring 2016 semester programme on Dynamics of Biologically Inspired Networks (https://mbi.osu.edu/programs/emphasis-programs/ previous-programs/spring-2016-dynamics-biologically-inspired-networks/) at the Mathematical Biosciences Institute, and numerous others.

\section{The articles in this issue}

We now provide a brief description of the articles in this special issue. One can find many of the key references in the burgeoning science of networks in the bibliographies of the papers that comprise the issue. We have also included a few of them in this introductory note (both above and below).

We open our special issue with a pair of articles on the study of "mesoscale structures" in networks. In such studies, which are among the most active topics in network science, one seeks to coarse-grain networks into intermediate-scale structures (e.g., collections of nodes) to try to infer properties such as circles of friends, functional groups in proteins, political voting blocs, and more. The first paper, by Gleich and Kloster [13], leverages a PageRank random walk [12] from a set of seed nodes to examine mesoscale network structures such as community structure and core-periphery structure. In the second paper, Cucuringu et al. propose and compare several novel techniques for studying core-periphery structure in networks [7]. A "community", which is the simplest type of mesoscale network structure, consists of a densely-connected set of nodes that is connected sparsely to other dense sets 
of nodes $[10,11,27]$. By contrast, "core-periphery structure" involves one or more cores of densely-connected nodes that also tend to be well-connected to peripheral nodes, but the peripheral nodes tend to be connected only sparsely to other nodes.

Another major topic in network science is the study of dynamical processes on networks, and several of the papers in our special issue address different aspects of this subject. One of the most important questions is to ask how nontrivial structural connectivity affects dynamical processes on networks [26]. For example, network structure can represent social contacts, and structural features can profoundly impact the propagation dynamics of diseases and memes [25]. Network architecture also has a huge effect on collective behaviour in networks of coupled oscillators [1,2] and many other phenomena. In the special issue's third paper, Do et al. [8] examine (as in the first two papers) how the interplay of microscale constituents leads to the emergence of macroscale properties. They exploit the network structure of Jacobian matrices from coupled dynamical systems to yield insights on phenomena such as collective behavior, and they illustrate their results using synchronization in a network of phase oscillators, including an "adaptive" [29] setting in which network connectivity itself is coupled to oscillator dynamics. Our fourth paper, by Coombes and Thul [6], extends the well-known "master stability function" (which can be used to determine when coupled oscillators exhibit synchrony) to networks of piecewise-smooth oscillators and then examines an application in neuroscience. When studying dynamical systems on networks, it is also crucial to consider how to control such dynamics [22]. The paper by Burbano L. et al. [5] analyses control strategies for achieving consensus in heterogeneous multi-agent systems.

Another important application of dynamical systems on networks concerns the spread of biological contagions, as illustrated in the paper by Vestergaard et al. [32], which examines the impact of spatially constrained sampling of temporal contact networks when evaluating the risk of epidemics. In addition to discussing a fascinating application, this paper considers several important issues in networks, such as data sampling (which is ubiquitous in the study of networks), time-dependent interactions, and spatial constraints. The importance of sampling in the study of networks is also examined in our penultimate paper, by Grindrod et al. [14], which takes an inverse-problem approach and studies an application to online brand allegiance in Twitter. Another central issue in networks is the study of important nodes, edges, and other substructures [23]. The study of so-called "central" (i.e., important) nodes in networks has an especially rich tradition in social network analysis [33]. In the final paper of our special issue, Schoch and Brandes [30] reconceptualize centrality, showing that some of the standard centrality measures can be cast into a unified framework based on semirings associated with walks on graphs (and bringing us back to the first paper in our special issue, which also considers walks on networks).

\section{Conclusions}

The study of networks is one of the most exciting areas of applied mathematics. It is prominent in numerous fields of scholarship - both theoretical and applied - and it interacts very closely with increasingly prominent applications and with data science. Our selection of papers are exemplars of numerous exciting applications, and we hope that our special issue serves as an invitation for readers to study networks of their own. 


\section{References}

[1] Arenas, A., Díaz-Guilera, A., Kurths, J., Moreno, Y. \& Zhou, C. (2008) Synchronization in complex networks. Phys. Rep. 469, 93-153.

[2] Ashwin, P., Coombes, S. \& Nicks, R. (2016) Mathematical frameworks for oscillatory network dynamics in neuroscience. J. Math Neurosci. 6, 2.

[3] Barthelemy, M. (2011) Spatial networks. Phys. Rep. 499, 1-101.

[4] Boccaletti, S., Bianconi, G., Criado, R., del Genio, C. I., Gómez-Gardeñes, J., Romance, M., Sendiña-Nadal, I., Wang, Z. \& Zanin, M. (2014) The structure and dynamics of multilayer networks. Phys. Rep. 544, 1-122.

[5] Burbano L., D. A., Delellis, P. \& di Bernardo, M. (2016) Self-tuning proportional integral control for consensus in heterogeneous multi-agent systems. Eur. J. Appl. Math. 27, 923-940.

[6] Coombes, S. \& Thul, R. (2016) Synchrony in networks of coupled non-smooth dynamical systems: Extending the master stability function. Eur. J. Appl. Math. 27, 904-922.

[7] Cucuringu, M., Rombach, P., Lee, S. H. \& Porter, M. A. (2016) Detection of core-periphery structure in networks using spectral methods and geodesic paths. Eur. J. Appl. Math. 27, 846-887.

[8] Do, A.-L., Boccaletti, S., Epperlein, J., Siegmund, S. \& Gross, T. (2016) Topological stability criteria for networking dynamical systems with Hermitian Jacobian. Eur. J. Appl. Math. 27, 888-903.

[9] Dorogovtsev, S. N., Goltsev, A. V. \& Mendes, J. F. F. (2008) Critical phenomena in complex networks. Rev. Mod. Phys. 80, 1275-1335.

[10] Fortunato, S. (2010) Community detection in graphs. Phys. Rep. 486, 75-174.

[11] Fortunato, S. \& Hric, D. (2016) Community detection in networks: A user guide. Phys. Rep., in press (arXiv:1608.00163).

[12] Gleich, D. F. (2015) PageRank beyond the Web. SIAM Rev. 57, 321-363.

[13] Gleich, D. F. \& Kloster, K. (2016) Seeded PageRank solution paths. Eur. J. Appl. Math. 27, 812-845.

[14] Grindrod, P., Higham, D. J., Laflin, P., Otley, A. \& Ward, J. A. (2016) Inverse network sampling to explore online brand allegiance. Eur. J. Appl. Math. 27, 958-970.

[15] Holme, P. (2015) Modern temporal network theory: A colloquium. Eur. Phys. J. B 88, 234.

[16] Holme, P. \& Saramäki, J. (2012) Temporal networks. Phys. Rep. 519, 97-125.

[17] JACKsON, M. O. (2010) Social and Economic Networks, Princeton University Press, Princeton, NJ, USA.

[18] JACKsON, M. O. \& Zenou, Y. (2014) Games on networks. In: P. Young \& S. Zamir (editors), Handbook of Game Theory with Economic Applications, Vol. 4, Elsevier, Amsterdam, The Netherlands, pp. 95-163.

[19] JaCobs, A. Z. \& Clauset, A. (2014) A unified view of generative models for networks: Models, methods, opportunities, and challenges. In: NIPS Workshop on Networks: From Graphs to Rich Data (arXiv:1411.4070).

[20] Kivelä, M., Arenas, A., Barthélemy, M., Gleeson, J. P., Moreno, Y. \& Porter, M. A. (2014) Multilayer networks. J. Complex Netw. 2, 203-271.

[21] KolaczyK, E. D. (2009) Statistical Analysis of Network Data: Methods and Notes, SpringerVerlag, Berlin, Germany.

[22] LiU, Y.-Y. \& Barabási, A.-L. (2016) Control principles of complex networks. Rev. Mod. Phys., 88, 035006.

[23] Lü, L., Chen, D., Ren, X.-L., Zhang, Q.-M., Zhang, Y.-C. \& Zhou, T. (2016) Vital nodes identification in complex networks. Phys. Rep., 650, 1-63.

[24] Newman, M. E. J. (2010) Networks: An Introduction, Oxford University Press, Oxford, UK.

[25] Pastor-Satorras, R., Castellano, C., Van Mieghem, P. \& Vespignani, A. (2015) Epidemic processes in complex networks. Rev. Mod. Phys. 87, 925-979. 
[26] Porter, M. A. \& Gleeson, J. P. (2016) Dynamical Systems on Networks: A Tutorial, Frontiers in Applied Dynamical Systems: Reviews and Tutorials, Vol. 4, Springer-Verlag, Heidelberg, Germany.

[27] Porter, M. A., Onnela, J.-P. \& Mucha, P. J. (2009) Communities in networks. Not. Am. Math. Soc. 56, 1082-1097, 1164-1166.

[28] Saberi, A. A. (2015) Recent advances in percolation theory and its applications. Phys. Rep. 578, $1-32$.

[29] Sayama, H., Pestov, I., Schmidt, J., Bush, B. J., Wong, C., Yamanoi, J. \& Gross, T. (2013) Modeling complex systems with adaptive networks. Comput. Math. Appl. 65, 1645-1664.

[30] Schoch, D. \& Brandes, U. (2016) Re-conceptualizing centrality in social networks. Eur. J. Appl. Math. 27, 971-985.

[31] Strogatz, S. H. (2001) Exploring complex networks. Nature 410, 268-276.

[32] Vestergaard, C. L., Valdano, E., Génois, M., Poletto, C., Colizza, V. \& Barrat, A. (2016) Impact of spatially constrained sampling of temporal contact networks on the evaluation of the epidemic risk. Eur. J. Appl. Math. 27, 941-957.

[33] Wasserman, S. \& Faust, K. (1994) Social Network Analysis: Methods and Applications, Cambridge University Press, Cambridge, UK. 